\title{
Non-linear signal analysis applied to surface wear condition monitoring in reciprocating sliding testing machines
}

\author{
Francisco Paulo Lépore Neto ${ }^{\mathrm{a}, *}$, José Daniel Biasoli de Mello ${ }^{\mathrm{b}}$ and Marcelo Braga dos Santos ${ }^{\mathrm{a}}$ \\ ${ }^{a}$ Mechanical System Laboratory, School of Mechanical Engineering, Federal University of Uberlandia, Av Joao \\ Naves de Avila 2121, Campus Santa Monica, Bloco 1M, Uberlandia, MG, 38400-902, Brazil \\ ${ }^{\mathrm{b}}$ Tribology and Materials Laboratory, School of Mechanical Engineering, Federal University of Uberlandia, Av \\ Joao Naves de Avila 2121, Campus Santa Monica, Bloco 1M, Uberlandia, MG, 38400-902, Brazil
}

\begin{abstract}
When the surfaces of two elastic bodies present relative motions under certain amount of contact pressure the mechanical system can be unstable. Experiments conducted on elastic bodies in contact shown that the dynamic system is self-excited by the non-linear behavior of the friction forces. The main objective of this paper is to estimate the friction force using the vibrations signals, measured on a reciprocating wear testing machine, by the proposed non-linear signal analysis formulation. In the proposed formulation the system global output is the sum of two outputs produced by a linear path associated in parallel with a non-linear path. This last path is a non-linear model that represents the friction force. Since the linear path can be identified by traditional signal analysis, the non-linear function can be evaluated by the global input/output relationships. Validation tests are conducted in a tribological system composed by a sphere in contact with and a prismatic body, which has an imposed harmonic motion. The global output force is simultaneously measured by a piezoelectric and by a piezoresistive load cells. The sphere and prismatic body vibrations are measured by a laser Doppler vibrometer and by an accelerometer respectively. All signals are digitalized at the same time base and the data is transferred to a microcomputer. The non-linear signal analysis technique uses this data to identify the friction force.
\end{abstract}

Keywords: Non-linear signal analysis, friction force identification, wear, vibration

\section{Introduction}

Nowadays the analysis of nonlinear dynamic systems and the analysis of nonlinear forces applied to linear systems are increasing of importance. These dynamic systems with nonlinear properties or time dependent properties cannot be analyzed using the theories developed to linear systems. Analysis of these kinds of systems demand complex methodologies to predict its response or even to estimate its dynamical characteristics and models parameters. Even the study of a linear systems could require a nonlinear signal analysis methodology, as shown by the following cases: Measuring nonlinear physical quantities using linear instrumentation; predicting the response of a linear system excited by a nonlinear force; and the behavior of a vibratory system subjected to friction force.

Classical signal analysis, used in the study of dynamical system, was developed to stationary random data. However, much of the random data of interest in engineering applications is nonstationary when viewed as a whole. Typical examples of nonstationary data are vibrations signals measured in dynamical systems subjected to nonlinear forces, in dynamical systems with temperature dependent physical parameters, signals obtained from dynamical

*Corresponding author. Tel.: +55 3432394148 Ext. 219; Fax: +55 34 32394206; E-mail: fplepore@mecanica.ufu.br. 
systems with cubic stiffness or time varying mass. Normally to analyze these kinds of signals the engineers use Volterra series to study the nonlinear and the time dependent properties [1].

However, Volterra series provide results that are difficult to be physically interpreted and are difficult to be calculated. Nevertheless these difficulties, it should be cited that the estimated system model is strongly dependent on the probability density functions (PDF) of the input signals applied to the dynamical system. Therefore, the outputs and the systems parameters obtained with inputs that don't have the same PDF nature cannot be compared.

Recently, a new methodology was developed to analyze nonlinear systems, based on multiple input/single output (MISO) linear analysis of a reverse dynamic system and on statistical relations between the input excitation data and the output response data. This methodology can estimate the nonlinear properties of the dynamical system when the input data and the output data are measured, or it can estimate the input from the previous knowledge of the systems properties and of the output data, or estimate the output if the input is measured. The main advantages of this inverse MISO nonlinear analysis methodology compared to the use of Volterra series are the low computational cost and the fact that the results are easy to be physically interpreted. Since the statistical nature of the PDF of input data is considered in the analysis the obtained results are statistically consistent [2].

A directly application of the nonlinear MISO analysis is the measurement of nonlinear forces acting on a linear system. Since the linear parameters of the instrumentation devices are well know, by means of traditional signal analysis and system identifications methodologies, the nonlinear force can be measured indirectly using the nonlinear MISO analysis applied to the output data. Using this methodology the dynamic system model and its parameters can be identified at the same time. Nonlinearities that can be represented as single-valued function, can be identified using the relations between the input and the output data probability distribution functions [2].

This paper presents a methodology to estimate the friction force, acting as excitation of a tribometer used to study the wear of materials, and also to analyze a vibratory mechanical system with a Coulomb damper, using the system linear properties and its output response.

The Coulomb law was chosen to model the friction due its validity in a wide range of tribological conditions [3]. This law of friction physical parameters can be easily interpreted. Additionally it should be noted that the nature of friction force do not change even when the values of the kinetic and static friction force changes [5].

To verify the behavior of the adopted law of the friction model, experiments were carried on a one-degree of freedom vibratory system with an attached Coulomb damper device. The experiments were conducted in three different tribological conditions: dry contact, solid lubrication, and lubricated with mineral oil.

To evaluate the efficacy of the proposed methodology, another group of experiments were conducted on a reciprocating tribometer used to study the solid lubrication performance of a thin Teflon layer, by means of the estimation of the friction force. The sensibility to small changes in the tribological conditions could be measured by the changes of the estimated friction force and are confirmed by the surface topography, obtained by an scanning electron microscope. This case study is very useful to tribology, since the researchers of this area believe that changes on the contact surface produces specific changes of the friction force [6]. Therefore the correct estimate of the friction force and its correlation with the surface wear, without influence of dynamical behavior of the tribometer, is an important application of the nonlinear signal analysis methodology [4].

\section{Fundamentals of non-linear signal analysis}

Physical parameters of a nonlinear system cannot be identified by traditional linear analysis since this technique requires that the probability density function of the inputs and outputs must have the same nature, condition not verified in nonlinear systems [1].

Volterra series theory can be used to represent nonlinear systems using sets of high order polynomial functions assembled in parallel. However, due the large calculation effort and dependencies of the Volterra series to the probability density function (PDF) of input data, Bendat [2] proposed a new methodology that uses the well-known theory of multiple input/multiple output linear systems (MISO) modified to be applied to signal analysis of non-linear systems.

The nonlinear signal analysis is based on the hypothesis that the nonlinear characteristics of the system is additive to its linear characteristics and that the nonlinear effects acting on one input produces instantaneous output. 


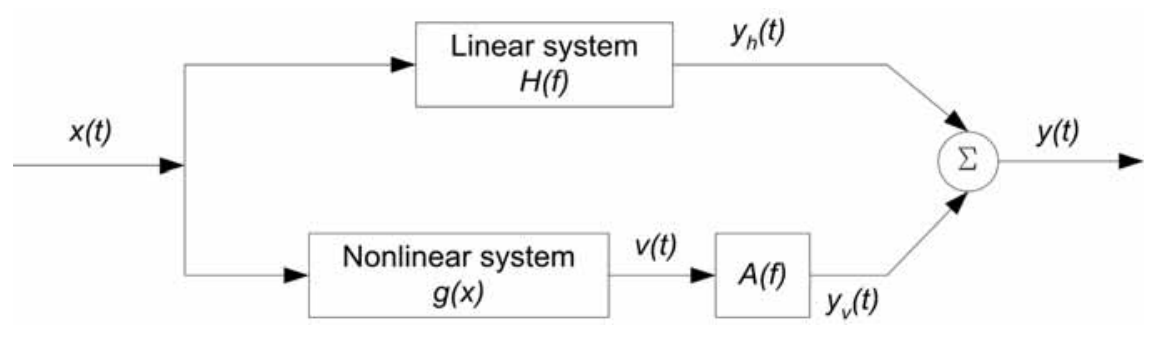

Fig. 1. Nonlinear MISO model block diagram.

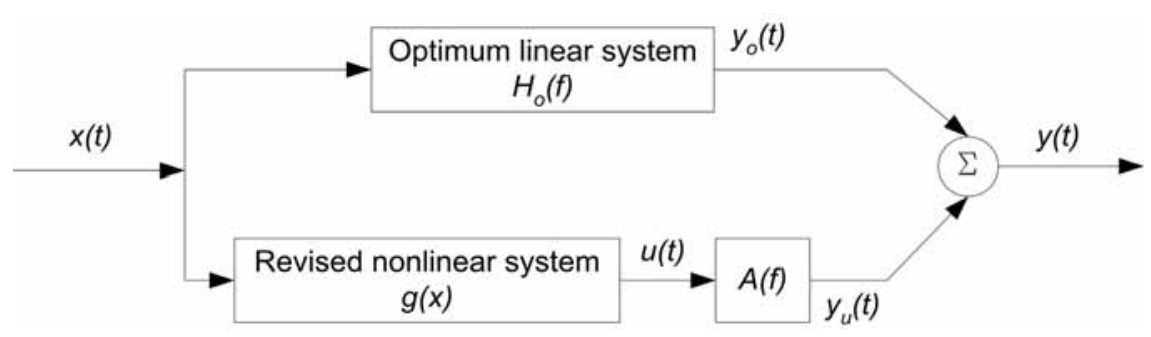

Fig. 2. Nonlinear MISO model with uncorrelated inputs.

The nonlinear system represented in the Fig. 1 do not have limitations regarding the characteristics of the signal input $x(t)$ PDF, as imposed by Volterra models. However to use this methodology a prior knowledge of the nonlinear functions $g(x)$ is required. However, Bendat [2] proposed that a prior analysis of the relation between cumulative probability function of the input data and the output data could estimate the nonlinear function $g(x)$.

On the non-linear MISO theory statistical uncorrelated inputs produce statistical uncorrelated outputs. This property can be used to separate the nonlinear and the linear parts of the total output $y(t)$. This implies that the coherence function between the nonlinear input $u(t)$ and the linear input $x(t)$ in the Fig. 2 is null at all frequencies. So, $y_{o}(t)$ and $y_{u}(t)$ are completely uncorrelated and represent, respectively, the outputs due to the linear and to the nonlinear input paths.

Calculating $v(t)=g(x(t))$ the power spectral densities and the coherence functions are determined by:

$$
\begin{array}{lll}
L_{x v}=\frac{S_{x v}}{S_{x x}} & S_{u y}=S_{v y}-L_{x v} * S_{x y} & H o=S_{x y} / S_{x x} \\
S_{y_{o} y_{o}}=\left|H_{o}\right|^{2} * S_{x x} & S_{u u}=S_{v v} *\left(1-\gamma_{x v}\right) & A=S_{u y} / S_{u u} \\
H=H_{o}-\left(S_{x y / S_{x x}}\right) * A & S_{y_{h} y_{h}}=|H|^{2} * S_{x x} & S_{y_{u} y_{u}}=|A|^{2} * S_{u u} \\
S_{y_{v} y_{v}}=|A|^{2} * S_{v v} & \gamma_{x v}=\left|S_{x y}\right|^{2} / S_{x x} * S_{v v} &
\end{array}
$$

The model represented in Figs 1 and 2 includes only one nonlinear function. However, it should be noted that this methodology can represent systems with more than one nonlinear behavior, by including additional paths. The PSD function and the coherence functions presented in Eq. (1) must be calculated considering all possible combinations of the parallel paths.

The use of nonlinear MISO formulation permits an easy correlation of the estimated parameters with physical properties of the assumed model of the nonlinear system even in models with more than one nonlinear property [2].

\section{Vibratory systems with nonlinear properties}

To apply the proposed methodology in the analysis of nonlinear dynamical systems should be done a prior mathematical and physical modeling to identify the linear and the nonlinear properties of this system. In the mechanical system shown in Fig. 3 the linear parameters $m, c$ and $k$ are respectively the mass, the viscous damping 


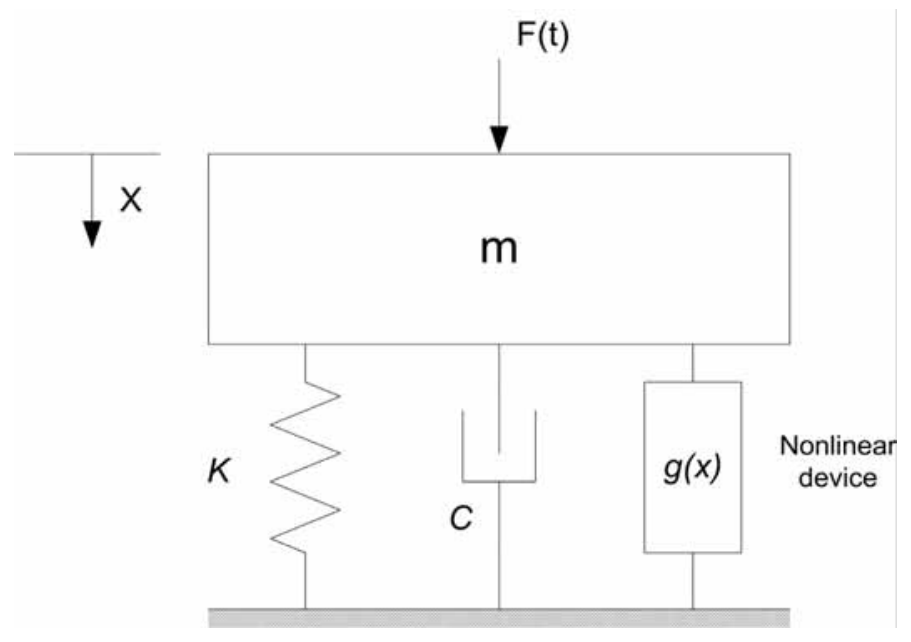

Fig. 3. One degree of freedom mechanical system with a nonlinear device.

and the suspension stiffness. A generic nonlinear device that produces a force $g(x)$ is connected in parallel to the suspension, and an external force $F(t)$ excites the system. This system time domain mathematical model is presented in Eq. (2) and the associated frequency domain model of Eq. (3) is obtained by taking the Fourier Transform of the Eq. (2).

$$
\begin{aligned}
& m \ddot{x}+c \dot{x}+k x+a g(x)=F(t) \\
& F(f)=[H] X(f)+A(f) F(g(x))
\end{aligned}
$$

The frequency dependencies of the nonlinear device are included in $A(f)$ and $F(g(x))$ is the Fourier transform of the nonlinear input $v(t)$. Equations (2) and (3) show that there are no imposed restrictions on the nature of the nonlinear function $g(x)$ and that the nonlinear and linear terms are additive, according MISO theory [2].

A generic linear system excited by a nonlinear force is represented in Fig. 4 and its mathematical model is given by Eq. (4).

$$
[m]_{n x n}\{\ddot{x}\}_{n x 1}+[c]_{n x n}\{\dot{x}\}_{n x 1}+[k]_{n x n}\{x\}_{n x 1}=\{F\}_{n x 1}
$$

The vector $\{F\}$ includes all nonlinear forces. Using the same notation of Eq. (2) the nonlinear force is given by Eq. (5), where $[A]_{n \times n}$ is diagonal matrix with non-zero elements only at the positions corresponding to the degrees of freedom where the nonlinear forces act. The same condition is verified for the vector $\{g(x)\}$ where nonlinear functions exist only at each corresponding degree of freedom.

$$
\{F\}_{n x 1}=-[A]_{n x n}\{g(x)\}_{n x 1}
$$

Assuming that the system is connected to the inertial reference by a spring and a damper, located at the degree of freedom " 1 ", representing for example, a load cell that connects this degree of freedom to the ground, Eqs (4) and (5) can be rewritten as follows.

$$
\begin{gathered}
{[m]_{n x n}\{\ddot{x}\}_{n x 1}+\left[c^{\prime}\right]_{n x n}\{\dot{x}\}_{n x 1}+\left[k^{\prime}\right]_{n x n}\{x\}_{n x 1}=-[A]_{n x n}\{g(x)\}_{n x 1}+\left\{F_{\text {load_cell }}\right\}} \\
\left\{F_{\text {load_cell }}\right\}=-\left[c^{\prime \prime}\right]_{n x n}\{\dot{x}\}_{n x 1}-\left[k^{\prime \prime}\right]_{n x n}\{x\}_{n x 1}
\end{gathered}
$$

Where:

$$
\begin{aligned}
{\left[c^{\prime}\right]_{n x n} } & =[c]_{n x n}-\left[c^{\prime \prime}\right]_{n x n} \\
{\left[k^{\prime}\right]_{n x n} } & =[k]_{n x n}-\left[k^{\prime \prime}\right]_{n x n} \\
{\left[c^{\prime \prime}\right]_{n x n} } & =\left[\begin{array}{lll}
c_{1} & 0 & 0 \\
0 & 0 & 0 \\
0 & 0 & 0
\end{array}\right]_{n x n}
\end{aligned}
$$




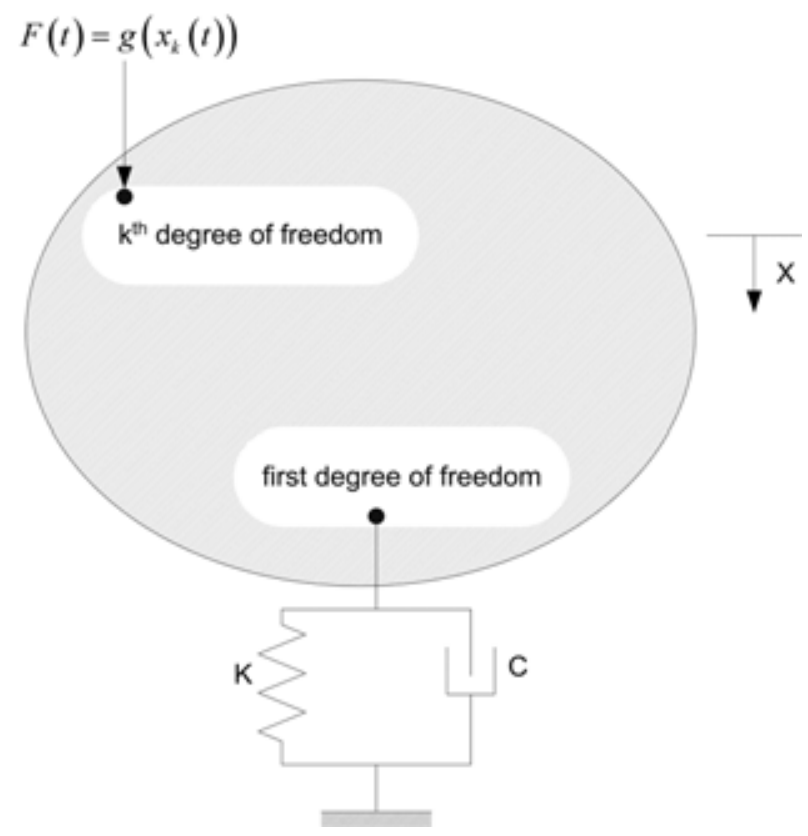

Fig. 4. Linear system subjected to a nonlinear force.

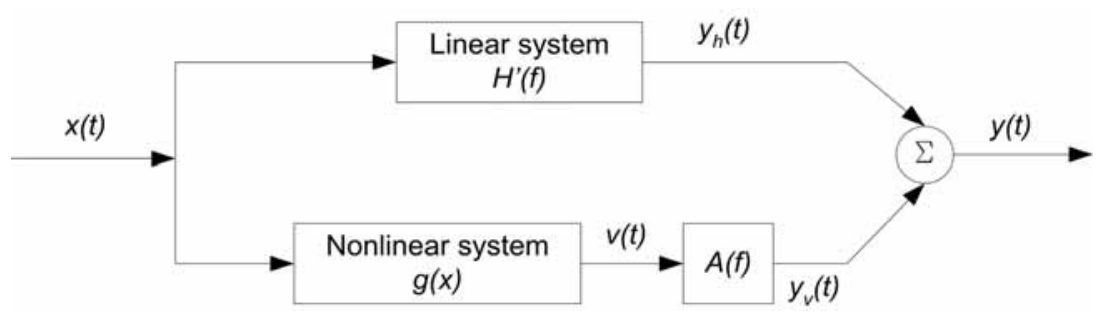

Fig. 5. MISO model used to determine the nonlinear force.

$$
\left[k^{\prime \prime}\right]_{n x n}=\left[\begin{array}{ccc}
k_{1} & 0 & 0 \\
0 & 0 & 0 \\
0 & 0 & 0
\end{array}\right]_{n x n}
$$

Isolating the force measured by the load cell at the right hand side of Eq. (6), the final representation of the system mathematical model results:

$$
[m]_{n x n}\{\ddot{x}\}_{n x 1}+\left[c^{\prime}\right]_{n x n}\{\dot{x}\}_{n x 1}+\left[k^{\prime}\right]_{n x n}\{x\}_{n x 1}+[A]_{n x n}\{g(x)\}_{n x 1}=\left\{F_{\text {load_cell }}\right\}
$$

The frequency domain representation of the linear system, subjected to nonlinear forces, is finally obtained by taking the Fourier transform on both sides of Eq. (8).

$$
\left\{F_{\text {load_cell }}(f)\right\}_{n x 1}=\left[H^{\prime}(f)\right]_{n x n}\{X(f)\}_{n x 1}+[A(f)]_{n x n}\left\{F\left(g\left(x_{k}\right)\right)\right\}_{n x 1}
$$

Using Eq. (9) and the concept presented in Fig. 1, the correct representation of a MISO system with nonlinear excitation force is represented in Fig. 5, where the matrix $[H]$ is replaced by $\left[H^{\prime}\right]$ to represent the FRF of the system of Fig. 4 with the terms $k_{1}$ and $c_{1}$ removed from stiffness and damping matrix and transferred to the right side of Eq. (8), representing the force measured by the load cell.

It should be noted that the force measured by the load cell includes the linear component $S_{y_{h} y_{h}}$ and also the nonlinear component $S_{y_{u} y_{u}}$. Moreover, to obtain the true nonlinear force, by means of nonlinear MISO signal analysis, a prior specification of the nonlinear function $g(x)$ is required. 
According to Eq. (9) and using the methodology explained in Section 2, the force measured by the load cell can be completely separated into a nonlinear parcel and into a linear parcel. So, the nonlinear excitation force is indirectly measured by $S_{y_{u} y_{u}}$ which is the estimate of the nonlinear term in the Eq. (1).

The same procedure can be applied to systems with several nonlinear effects without loss of generality. In this paper, the formulation is applied to identify the nonlinear force acting on a linear system, using the measured system response. The analysis of linear systems submitted to friction forces are of great interest to tribology field of research, since in the wear experiments the friction force between the specimens carries information about the surface condition [3].

\section{Nonlinear model of the friction force}

The proposed model for the friction force between two surfaces is capable to represent stick and slip displacements and also its hysteretic nature. The Coulomb friction law is used to relate the normal and tangential forces at the contact surfaces. Figure 6 presents the physical behavior of the contact between one static surface and a second body during one cycle of harmonic imposed displacement. The mathematical model is given by Eq. (10) for each kinematics condition at the contact.

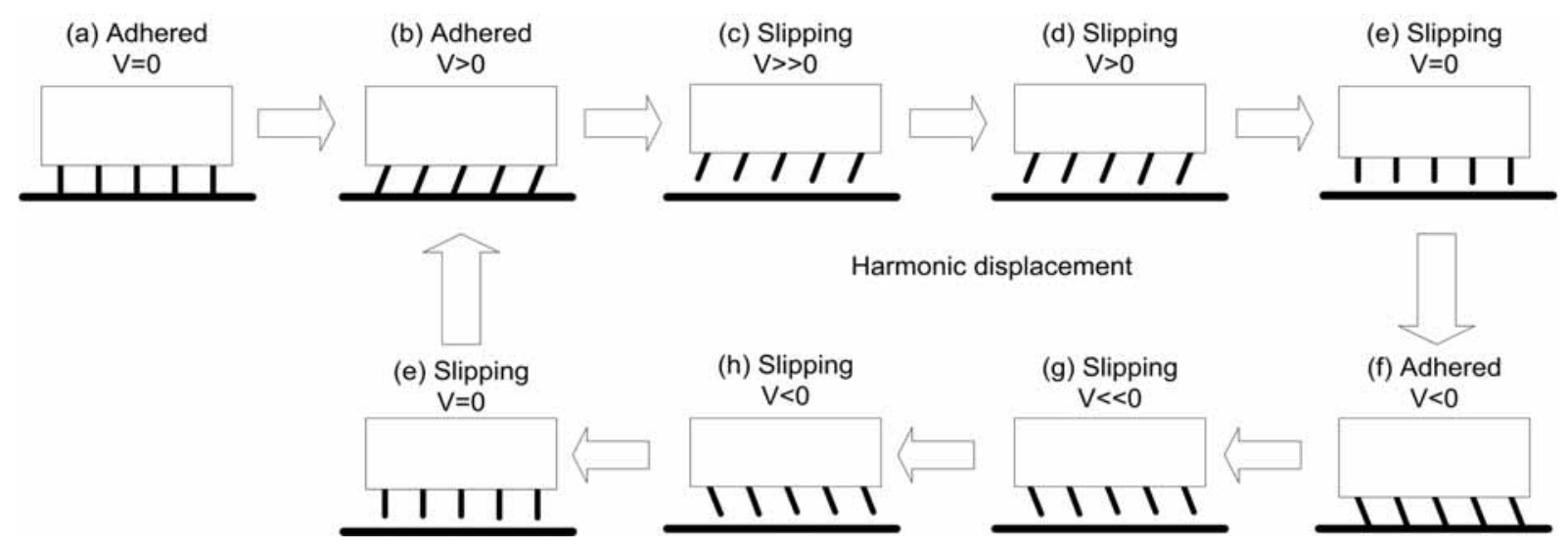

Fig. 6. Schematic diagram of the friction model.

If the body is at rest and will start the movement

$F_{\text {Coulomb }}=\operatorname{sgn}\left(v_{\text {rel }}\right) * u_{\text {rel }} * K_{\text {Coulomb }}$

If the body is slipping in the static surface

$F_{\text {Coulomb }}=\operatorname{sgn}\left(v_{\text {rel }}\right) * F_{\text {Coulomb }}$

$u_{s}=u_{\text {rel }}$

If the body is adhered to surface after its slip in the static surface

$$
F_{\text {Coulomb }}=\operatorname{sgn}\left(v_{\text {rel }}\right) *\left(u_{\text {rel }}-u_{s}\right) * K_{\text {Coulomb }}
$$

The slip displacement $u_{s}$ do not change if the body is adhered to the static surface. The friction stiffness, related to elastic deformation of the asperities in contact, is defined as $K_{\text {Coulomb. The relative velocity and displacement }}$ are defined as $v_{\text {rel }}$ and $u_{\text {rel }}$, respectively.

Using this model, the nonlinear function $g(x)$ of the MISO analysis, is completely defined. It can be verified that $F_{\text {Coulomb }}$ is function only of the state variables of the global system [4]. 

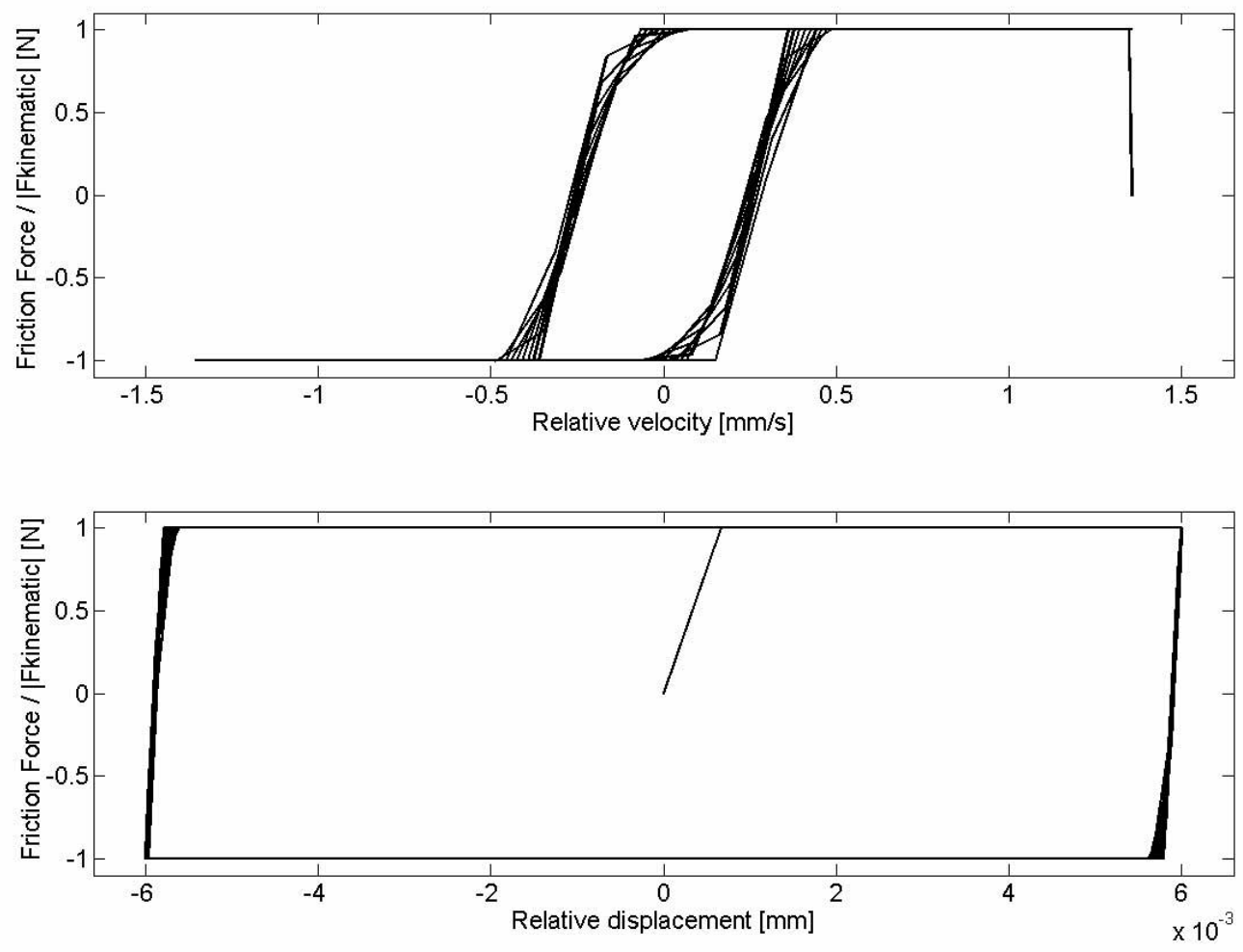

Fig. 7. Behavior of the nonlinear friction model.

Imposing $u_{\text {rel }}(t)$ as a sinusoidal displacement on Eq. (10), the normalized the friction force behavior is calculated and presented by Fig. 7.

The hysteretic and chaotic natures of the friction are evident. Comparing Figs 6 and 7 it should be noted that the elastic potential energy, stored in the deformed asperities of the contact, is not released until the body and the surface adhere again. This condition is different at the start of movement, when the adhesion between the body and the surface occurs without any previously stored potential energy. Hysteresis is represented by transition from stage " $h$ " to stage " $b$ " and from stage " $d$ " to stage " $f$ ", as shown in the Fig. 6.

\section{Results and discussion}

The proposed nonlinear MISO signal analysis methodology is used to provide the best estimate of the friction force that occur in the following two classes of experimental tests.

A. One degree of freedom linear vibratory system with a Coulomb friction damper: In this group of experiments the vibratory system was excited by an harmonic force. The friction force is obtained by MISO nonlinear signal analysis, and compared with the force signal measured by a load cell installed between the mass and the friction damper;

B. Reciprocating wear test of an aluminum specimen covered with a thin Teflon ${ }^{\mathrm{TM}}$ layer: This group of experiments was conducted to verify if the friction force, estimated by MISO nonlinear signal analysis, is sensitive to changes on the tribological conditions during the wear tests. 
The experiment conducted with a linear vibratory system with a Coulomb damper attached was used to confirm the validity of adopted Coulomb law of friction. Since the linear parameters of the vibratory system are easily determined by traditional linear signal analysis, the nonlinear influence caused by the Coulomb damper can be identified.

The second group of experiment was used to show the sensitivity of the estimated friction force to changes of the tribological condition of the mechanical contact. Small changes of the friction force amplitude are correlated with the wear of Teflon ${ }^{\mathrm{TM}}$ layer. The Teflon ${ }^{\mathrm{TM}}$ wear the friction force increases gradually until the Teflon ${ }^{\mathrm{TM}}$ layer was completely removed from the contact area. So this experiment is suitable to cover a wide range of changes in the amplitude of fiction force.

\subsection{Case A: One degree of freedom vibratory system with a Coulomb damper}

The experimental apparatus shown in Fig. 8 is composed by a rigid aluminum plate mounted on an elastic suspension consisting of four parallel elastic beams. The suspension geometry along with the large difference between the moments of inertia of the beams cross sections produce an one degree of freedom vibratory system at the frequency band bellow $100 \mathrm{~Hz}$. It is used to validate the adopted model for the friction force. The load cell 02 , located between the table and the Coulomb damper, measures the friction force. However, the signal measured by this sensor is not the actual friction force, since it includes the inertia force generated by the moving mass of the Coulomb damper.

The nonlinear system model represented by Fig. 9 is correlated to experimental apparatus as follow:

- Linear system: Vibratory system, where $m$ is the moving mass, $k$ is the suspension stiffness and $c$ is the viscous damping coefficient;

- Nonlinear system: Coulomb damper installed between $m$ and the fixed 'rigid' support. It consists of an Ushaped steel structure with four bronze pads and a central rigid plate, as shown by the left part of Fig. 8. The normal contact force can be adjusted by a screw with an helical spring that compress the pads against the central plate.

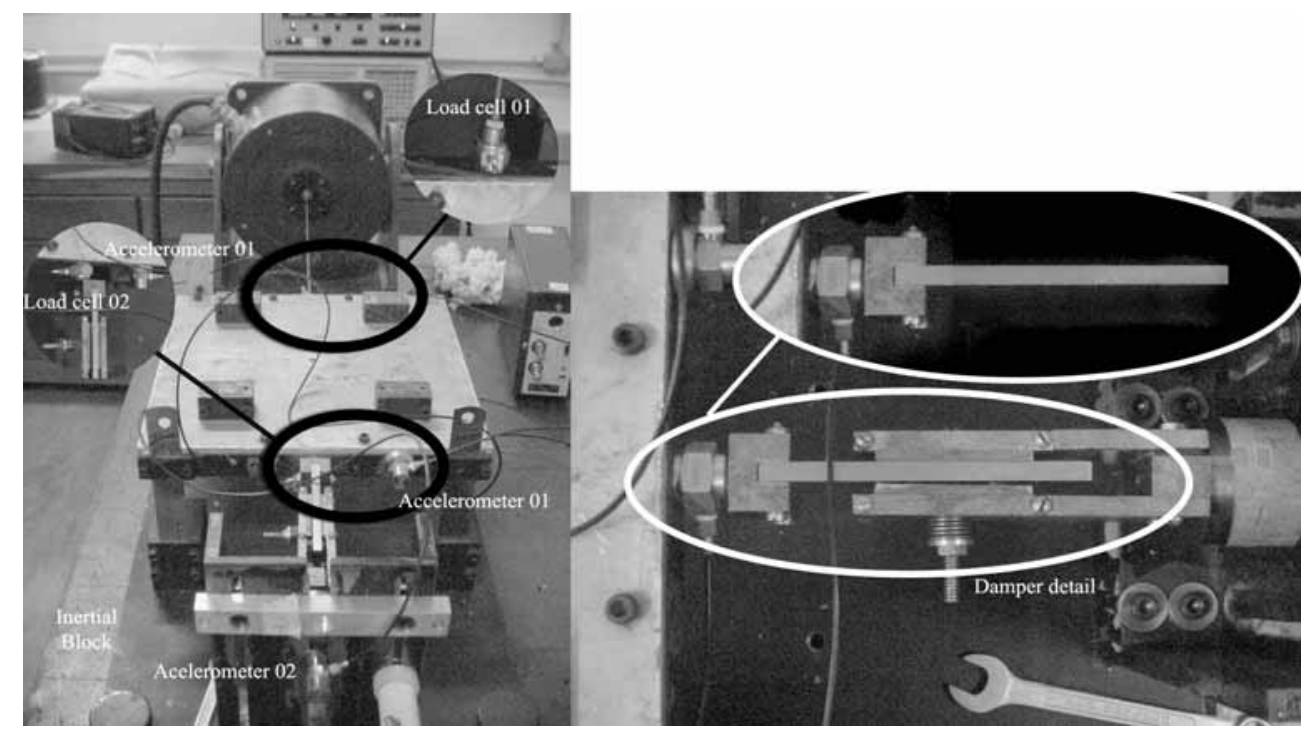

Fig. 8. Single degree of freedon vibratory system experimental set up.

The table movement is measured by the accelerometer 01 , and the accelerometer 02 measures the small, but possible, displacement of damper support. Load cell 01 measures the excitation force, generated by an eletrodynamic shaker, and load cell 02 measures the force due Coulomb friction damper plus the inertia force of its moving part. The nonlinear MISO system model can be configured as follows: 


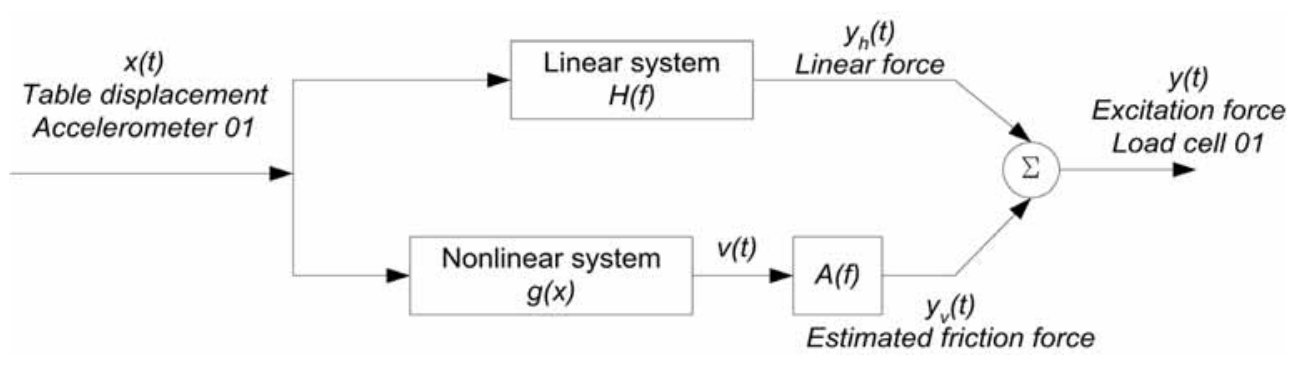

Fig. 9. Vibratory system nonlinear model.

To validate both the nonlinear MISO formulation and the Coulomb friction law, experiments were conducted with harmonic excitation forces at $10 \mathrm{~Hz}$, which is bellow the vibratory system natural frequency, and at $18 \mathrm{~Hz}$, which is very close to the system natural frequency.

The lower frequency was chosen to assure that the force measured by load cell 02 produce the best approximation to the real friction force, minimizing the effect caused by the inertia force of the damper moving part and the vibrations amplitudes of the fixed support. The relation $|\mathrm{F} / \mathrm{A}|$ between the force, measured by the load cell 02 , and the acceleration, measured by accelerometer 01, was estimated applying a white noise excitation to the vibratory system, removing the U-shaped structure of the damper. The transfer function shown by Fig. 10, at the 5-70 $\mathrm{Hz}$ frequency band, indicates constant value of the F/A magnitude, and null phase difference. The damper moving mass was calculated and results equal to $0.2374 \mathrm{Kg}$. In this case, the inertia force effect, calculated using the second Newton's law, can be directly subtracted from the overall force measured by load cell 02 in the experiments with the complete damper installed.

At frequencies above $70 \mathrm{~Hz}$ the set up of the load cell and the damper moving part creates a vibratory system with additional axial and flexural modes, modifying the global force measured by the load cell. Therefore, when frequency increases, the friction force becomes very small compared to the other dynamic forces measured by the load cell 02, which are proportional to the square of the frequency. This behavior can be observed on the frequency spectra of the estimated and measured friction force presented by Figs 11(a) and 11(b). An amplitude modulation effect is observed in Fig. 11(b), generated by the motion of the large inertial block, upon which the vibratory system is mounted, which has one of its natural frequencies near to $2.5 \mathrm{~Hz}$.

At the excitation frequencies there are reasonable agreement between the global force measured by the load cell 02 and the estimated friction force. Figure 11 (a) shows, at $10 \mathrm{~Hz}$, an amplitude difference of only $3.5 \%$, and from Fig. 11(b), the difference is $30 \%$ at $18 \mathrm{~Hz}$. The larger difference at $18 \mathrm{~Hz}$ is due mainly to the inertial force effect as explained before.

These two experiments show that at frequencies other than that of the excitation, the differences between the estimate friction force and the global measured force are very high. A first source of error is related to the dynamics of the set up of the load cell and the damper moving part. Another source of error is the adopted nonlinear function $g(x)$ presented by Eq. (10). For this model, the theoretical friction force is assumed to be a square wave, with small modification to include the hysteretic and the contact stiffness effects, but it does not consider other dynamic effects, which can exist in the real friction force or in linear system dynamics, including the load cell itself.

Using the time domain signals, the estimated friction force and the normalized global force measured by load cell 02 are plotted against the relative velocity, VR, measured between the two ends of the damper device, for excitations at $10 \mathrm{~Hz}$ and $18 \mathrm{~Hz}$.

Several cycles of the signals are shown in Fig. 12. The main differences between the estimated and measured friction force occur at $|\mathrm{VR}| \geqslant 0.01 \mathrm{~m} / \mathrm{s}$, indicated by the side lobes which are generated by the inertia effect on the measured global force. For values of $|\mathrm{VR}|<0.01 \mathrm{~m} / \mathrm{s}$ the estimated and measured cycles present almost the same slope.

Analyzing the relation between the friction force and the relative displacement at the adhered region, the tangential contact stiffness can be estimated, resulting $K_{\text {Coulomb }}=1.610^{6} \mathrm{~N} / \mathrm{m}$.

However, the proposed model is capable to represent the main effects, including stick and slip phenomenon, and permits a good approximation to the friction force, modeled by the Coulomb law. Inertia effects are responsible by 

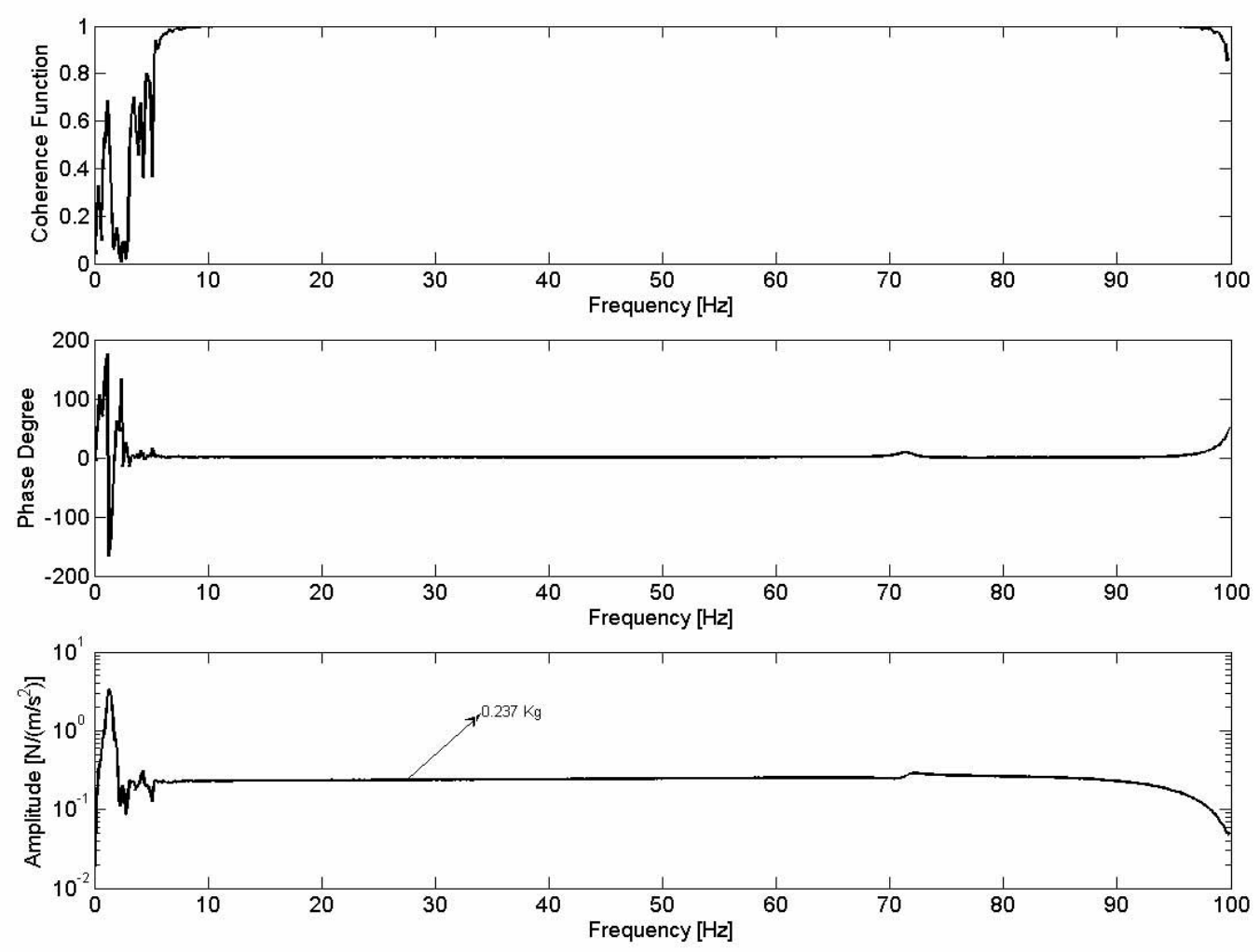

Fig. 10. Transfer function between the signals from load cell 02 and the accelerometer 01 .
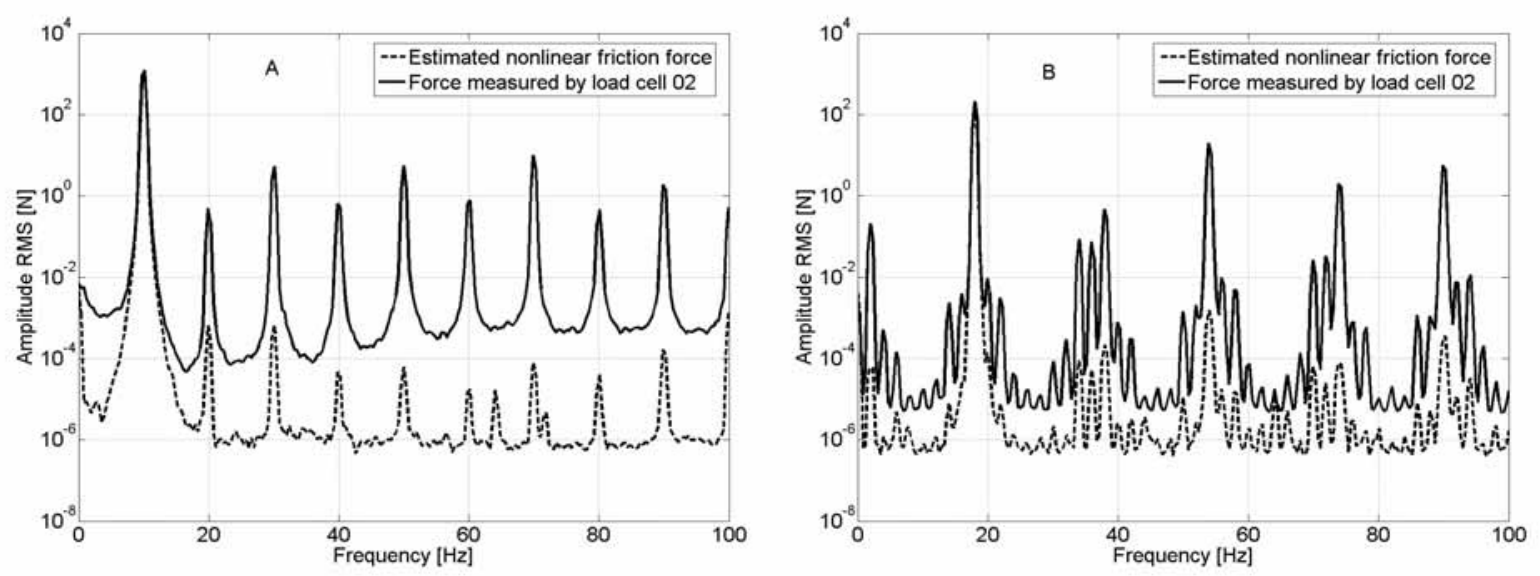

Fig. 11. Global and estimated force spectral densities: (a) at $10 \mathrm{~Hz}$ excitation and (b) at 18 excitation.

the large difference present at higher frequencies, but they were identified by the proposed methodology, using the low frequency experiments.

The estimates of the static and kinematic friction coefficients cannot be calculated without the knowledge of the 

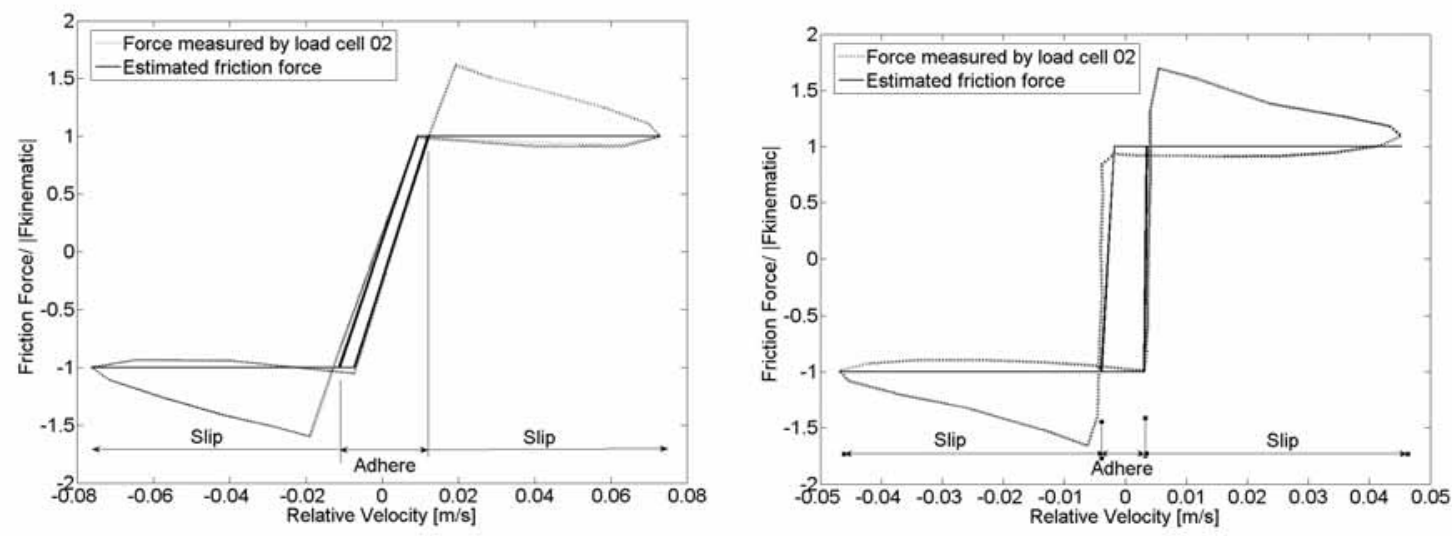

Fig. 12. Normalized global and estimated force vs. relative velocity: (a) at $10 \mathrm{~Hz}$ excitation and (b) at $18 \mathrm{~Hz}$ excitation.

normal force applied at the bronze pads. If the normal force signal is measured, its frequency spectrum can be used to calculate the friction coefficients.

Other friction force models can be assumed and the proposed methodology can be successfully applied. However, complex models that use large number of parameters can be identified, but the physical interpretation of the results may be very difficult.

\subsection{Case B: Reciprocating wear test in one specimen of aluminum covered by a thin Teflon ${ }^{\mathrm{TM}}$ layer}

Experiments with the reciprocating wear test machine were conducted using the formulation presented by Eq. (9) and the nonlinear force model presented by Eq. (10). The experimental set up, the instrumentation chain and some of the test conditions are presented by Fig. 13. The tribometer is an equipament PLINT\&PARTNERS CO - UK, model TE67/8834. It has an horizontal guided table driven by a motorized crank-shaft mechanism. The table harmonic motion amplitude and frequency can be set by the electric motor speed and by the excentricity of the mechanism. The aluminum specimen is installed on the table and a sphere, located at the end of a guided rigid shaft, applies a normal force at the contact. The normal force value is set by dead weights installed on the rigid shaft. A rigid pivoted lever connects the sphere shaft to the measuring load cell.

Based on Fig. 13 and Eq. (9) the block diagram associated with the nonlinear MISO system is constructed and presented in Fig. 14. The linear system represents the path from the sphere up to the piezoelectric load cell. The nonlinear path represents the friction force and the mechanical gain of the rigid lever. The $g(x)$ nonlinear function is expressed at the reference frame fixed to the reciprocating aluminum specimen

The reciprocating wear machine experiments take until 3 hours to complete. The signals are simultaneously acquired at $10 \mathrm{kSamples} / \mathrm{s}$, using a 16 channel and 12 bits $\mathrm{AD}$ converter. The frequency spectrum averages of 10 blocks with 32768 points each, are stored in a hard disk at 60 seconds intervals, generating the data set related to the evolution of wear along the test duration.

After completion of each test, the specimens were removed from the reciprocating machine and analyzed in a scanning electron microscope (SEM) to compare their surface conditions and the predominant wear mechanism with the evolution of the friction force estimated by the nonlinear MISO technique.

Three classes of experiments were carried out regarding the final surface condition of the aluminum specimen: I) the experiment was stopped before any damage occur to the Teflon ${ }^{\mathrm{TM}}$ layer; II) the experiment was stopped when the layer degradation is minimum; III) the experiment was conducted until the Teflon ${ }^{\mathrm{TM}}$ layer was completely removed.

The results obtained with nonlinear analysis, shown by the following figures, express the RMS value of the estimated friction force as function of the experiment time, These RMS values were calculated at the frequency band of $0-10 \mathrm{~Hz}$, where the coherence function between the input and the output data has near unit values.

Figure 15 shows that the friction force RMS amplitude presents small oscillations around $0.23 \mathrm{~N}$. the picture of the surface, taken after 1080 seconds, has no visible degradation. The result from the experiment of Fig. 16 repeats 


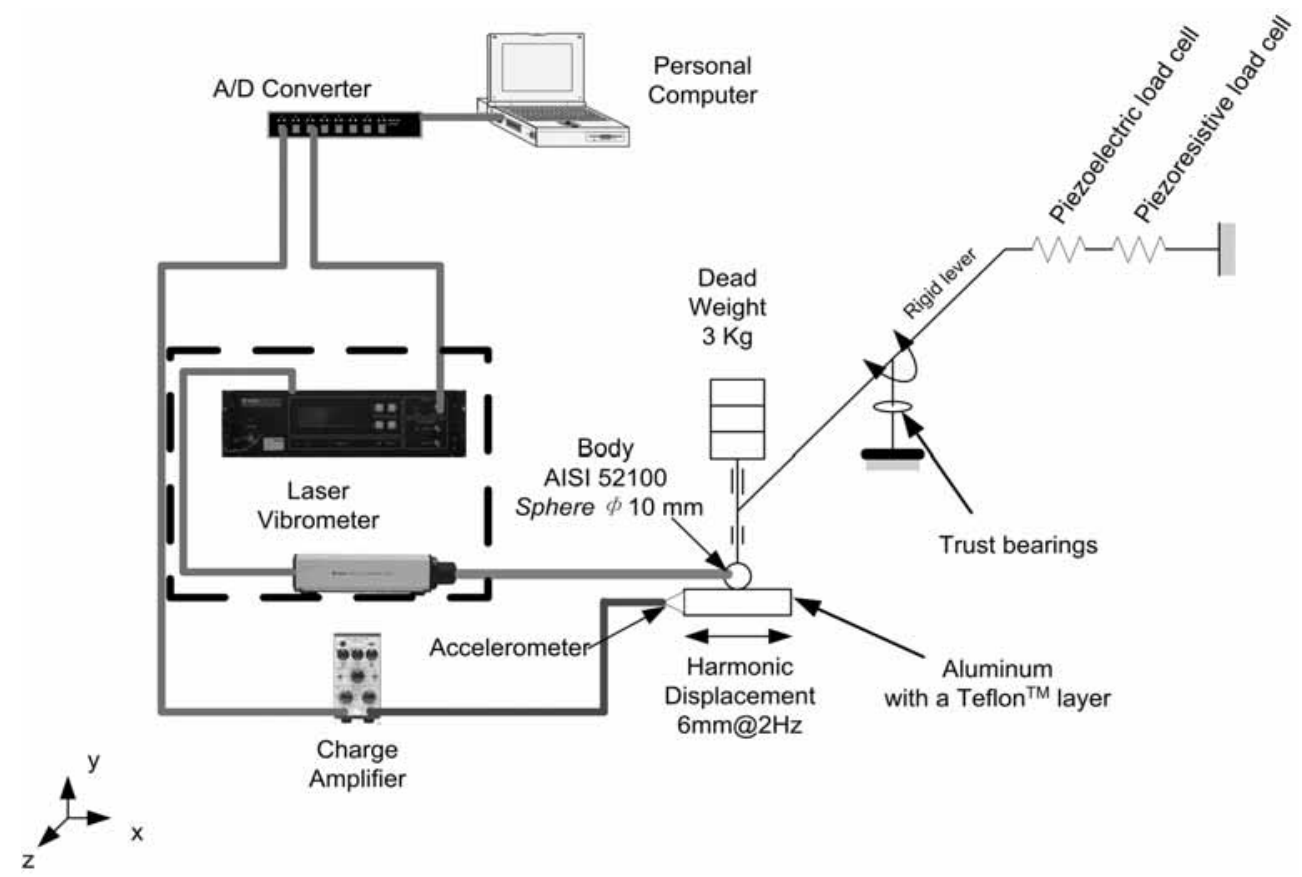

Fig. 13. Schematic diagram of the tribometer and the instrumentation chain.

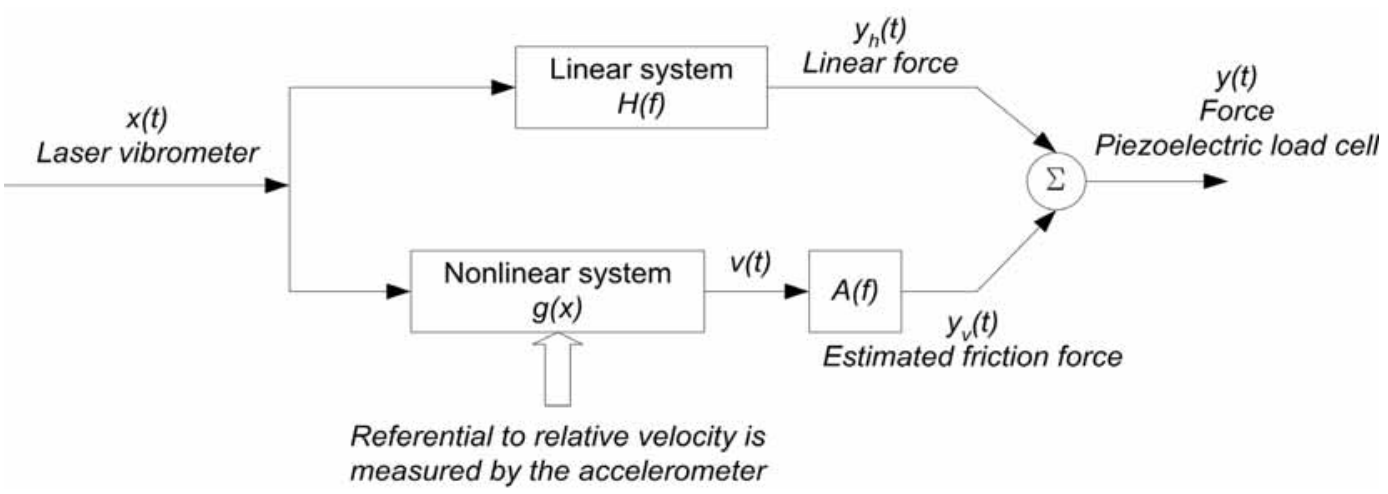

Fig. 14. Wear tests experiment nonlinear MISO model.

the same behavior for time less than 1080 seconds, as the previous one, but shows an increase of the force amplitudes from experiment time around 1200 seconds, reaching a maximum value at 4500 seconds. After this time the force reduces. This behavior is correlated to the surface conditions, indicated by the formation of some cracks on the Teflon ${ }^{\mathrm{TM}}$ layer, followed by its fragmentation promoted by the contact force.

The experiment with longest duration is shown by Fig. 17 and is consistent with the other two. After 6000 seconds the layer fragmentation continues and the force presents spikes that are related to the presence of Teflon ${ }^{\mathrm{TM}}{ }_{\mathrm{small}}$ particles that remains in the contact region, but some contact between the sphere and the aluminum substrate begins to occur. After 9000 seconds the RMS values of the estimated friction force presents large spikes that seems to be related with the contact between the sphere and the aluminum, indicating that the Teflon ${ }^{\mathrm{TM}}$ layer was completely removed. There is a first important tribological event at 1200 seconds and a second between 6000 and 8000 seconds and a third event after 9000 seconds, proved by the changes of the friction force values and also by the SEM photography of the specimen surface. 

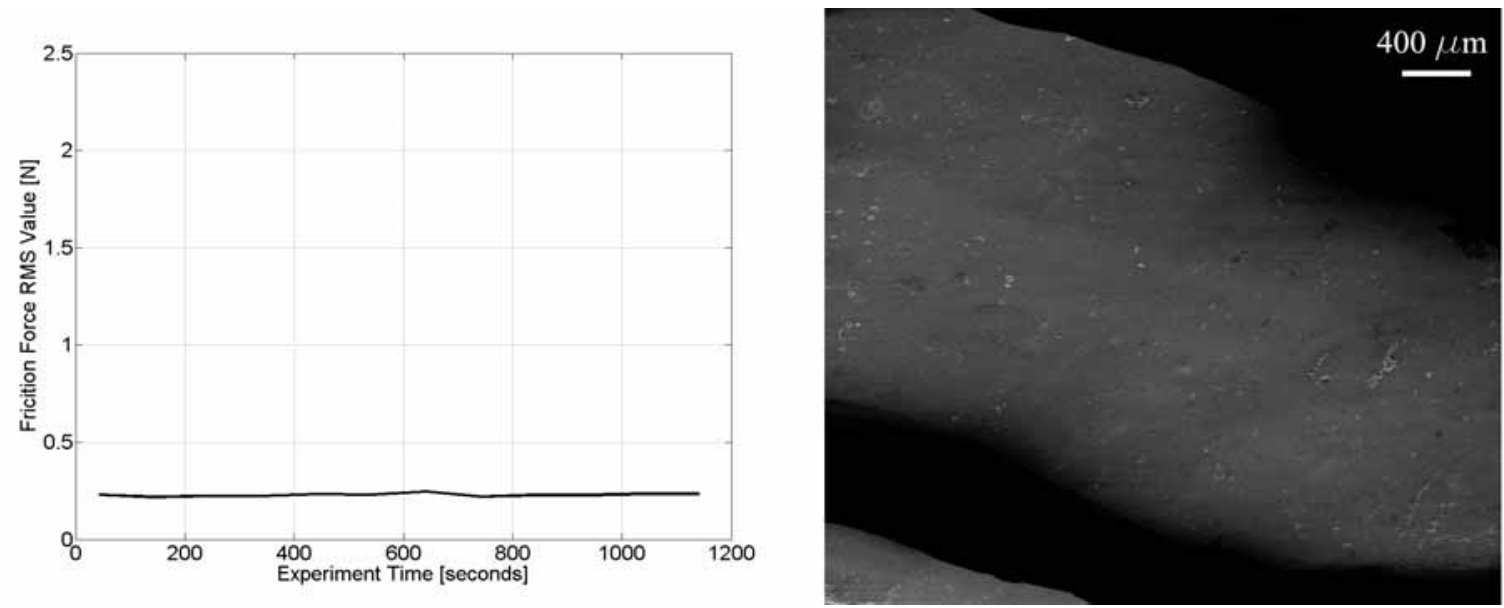

Fig. 15. Estimated friction force and surface SEM photography: Class I experiment.
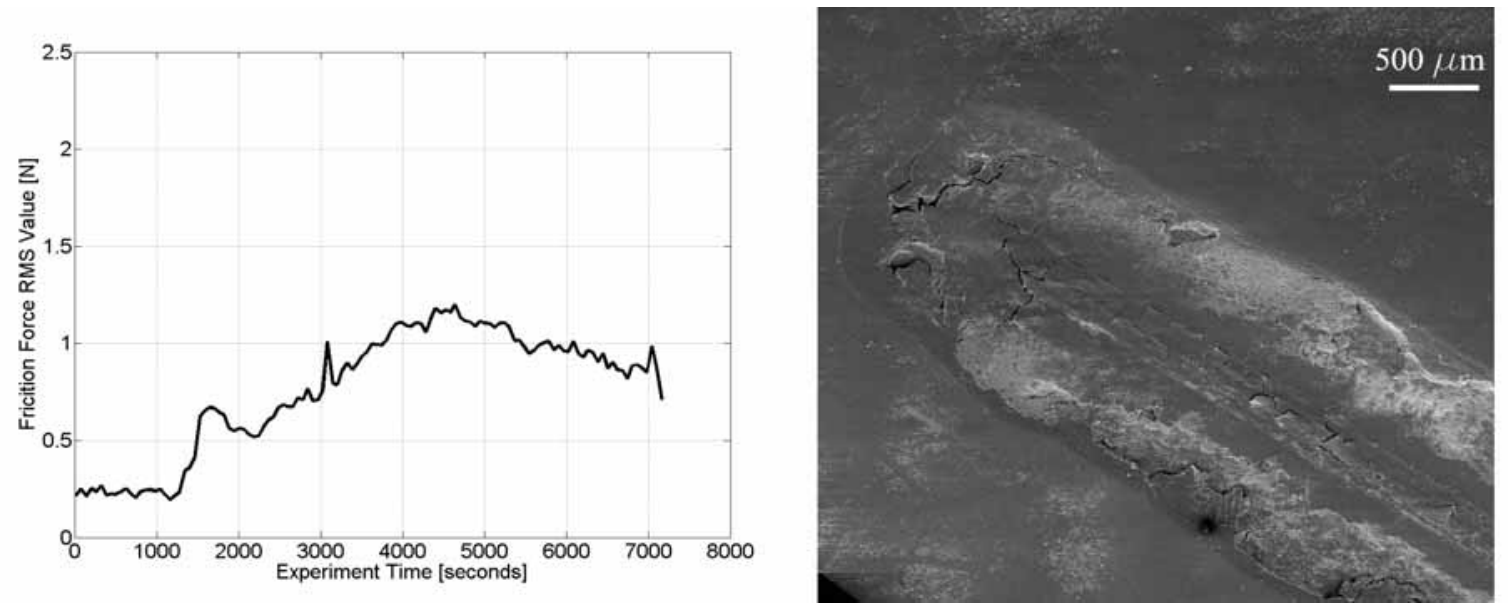

Fig. 16. Estimated friction force and surface SEM photography: Class II experiment.
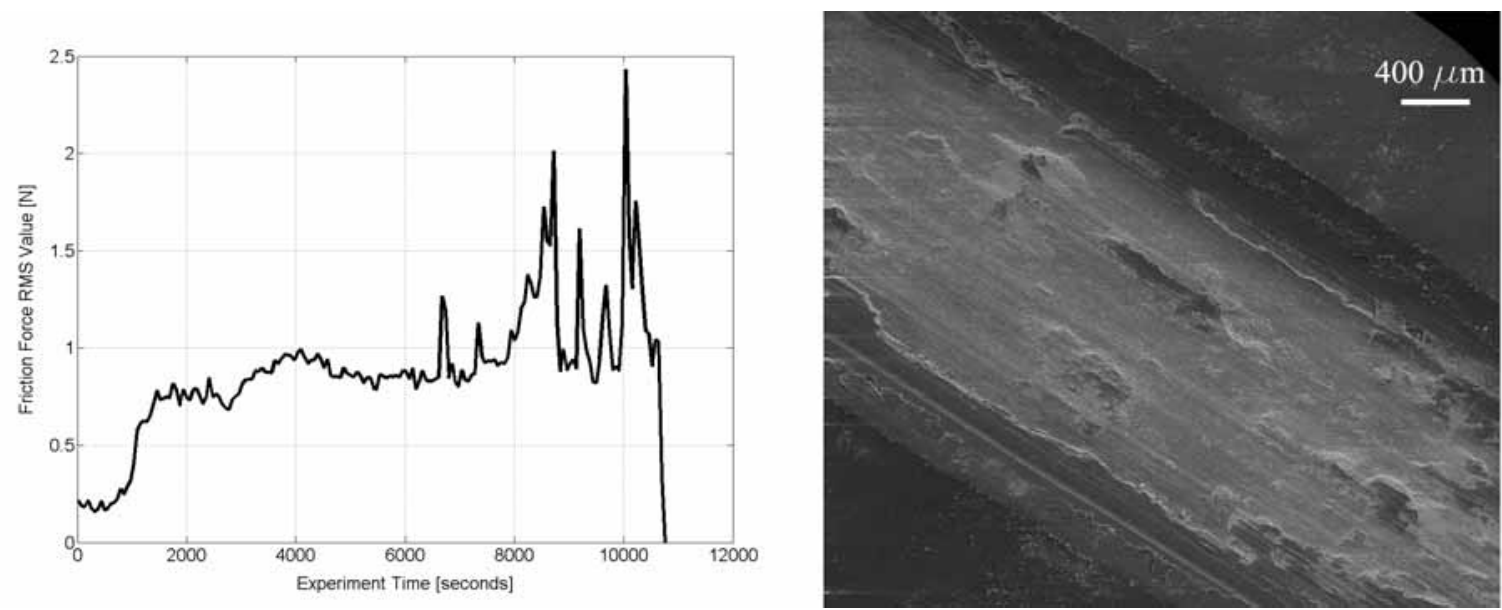

Fig. 17. Estimated friction force and surface SEM photography: Class III experiment. 


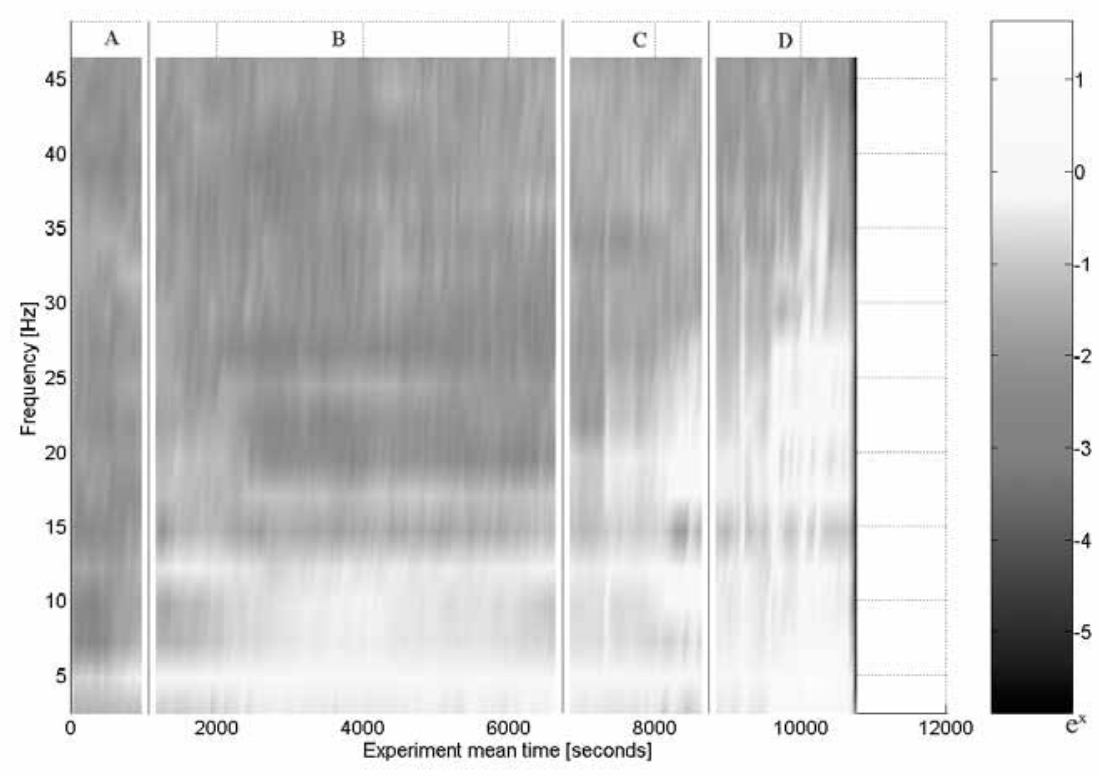

Fig. 18. Comparison of the Global and estimated forces from Class III experiment.

The estimated friction force is sensible to small changes in real friction force amplitudes. This is an important tool to design tribological wear studies. Performing a first long duration experiment and monitoring the estimate of the friction force it is possible to determine when the tribological events occur. This way, the interruption of each short time experiment can be planned in order to submit the specimen to a SEM analysis. So the proposed methodology was useful to correlate the wear mechanisms with a friction force signature. Since this signature was associated with specific wear events it will be possible to observe the wear evolution during other tribological tests with an aluminum substrate covered by a Teflon ${ }^{\mathrm{TM}}$ layer.

\section{Conclusion}

The proposed methodology was firstly used to validate the friction Coulomb law using a discrete damper device installed on a single degree of freedom vibratory system. The measurements of the global force and of the relative motion at the damper device extremities were compared to the friction force estimated by an inverse MISO model. This model has a linear path representing vibratory system dynamics and a nonlinear path containing a modified Coulomb friction law model that includes the tangential contact stiffness and the stick-slip effect. The best agreement between the global force measured by the load cell 02 and the estimated friction force is obtained at low excitation frequencies. This has occurred because the load cell stiffness and the mass of the moving part of the damper device make up a dynamic system that modifies the global force measurement. At higher excitation frequencies, the friction force amplitude remains constant, but mainly the inertia force increases with the square of the frequency. This effect is not a limitation of the proposed methodology. It only indicates that the load cell 02 cannot be used to directly measure the friction force. The results presented by Figs 11 and 12 show that the adopted friction force model is suitable to represent the stick and the slip behavior and also to identify the force amplitude and the contact tangential stiffness. The values of the friction static and kinematic coefficients were not calculated, since the normal contact force was not measured. Other friction force models can be assumed and the proposed nonlinear MISO methodology can be successfully applied.

The second set of experiments shown the efficacy of the methodology to estimate the friction force on a tribological wear test, without the influence of the tribometer dynamics. The modified Coulomb friction model is the same used 
before, but in this case the objective was to monitor the friction force evolution along the experiment time, in order to verify the force sensitivity with respect to the surface wear condition. The results presented by Figs 15, 16 and 17 clearly indicate the transition of the wear mechanisms associated with the changes of the estimated friction force. This characteristic is useful to plan the wear tests stop conditions in order to make a deep analysis of the surface using SEM microscopy. Another important feature is the possibility to compare the friction force estimates calculated in wear experiments executed with different settings on different tribometers. In any case, since the friction force is identified, static and kinetic friction coefficients can be calculated if the normal force between two bodies are measured, or other modeled parameters can be adjusted. The choice of the $0-10 \mathrm{~Hz}$ frequency band to analyze the measured signals was dictated by the excitation frequency imposed by the tribometer.

The obtained results suggest that the analysis and the identification of a general nonlinear dynamic system are feasible by using of the proposed nonlinear inverse MISO technique. This methodology can also be used to identify nonlinear effects that exist on a linear system, without any restrictions imposed on the statistical nature of the measured signals. If the nonlinear behavior is well modeled, the methodology is capable to identify and separate the exact nonlinear part from the global response of the system. In the case of mechanical systems with known linear properties the inverse MISO representation is simple and easy to implement and the estimated results can be physically interpreted. Since no restrictions are imposed on the nature of the assumed model of the nonlinear functions, the identification of physical parameters of highly nonlinear mechanical systems can be done.

\section{Acknowledgement}

The authors acknowledge the financial support provided by CNPq.

\section{References}

[1] J.S. Bendat, P.A. Palo and R.N. Coppolino, A general indentification technique for non-linear differential equations of motions, Probabilist Engineering Mechanics 7 (1992), 43-61.

[2] J.S. Bendat, Nonlinear Systems Techiniques and Applications, John Wiley and Sons Inc., New York, 1997.

[3] I.M. Hutchings, Tribology Friction and Wear of Engineering Materials, CRC Press Inc., Boca Raton, USA, 273.

[4] F.P. Lépore Neto, J.D.B. Mello and M.B. Santos, Correlação entre a força de atrito obtida através de análise de sinais não linear e o desgaste em um tribômetro do tipo alternativo, In: 59 Annual Congress - ABM International, 2004, São Paulo. 59 Annual Congress International. São Paulo, ABM Brasil, 2004. 4434-4443.

[5] S.L.R.H. Nowotny and S.F. Wayne, The role of specimen stiffness in sliding and impact wear, Wear 77(1,15) (March 1982), 13-28.

[6] J.A. Williams, Engineering Tribology, Oxford University Press, UK. 

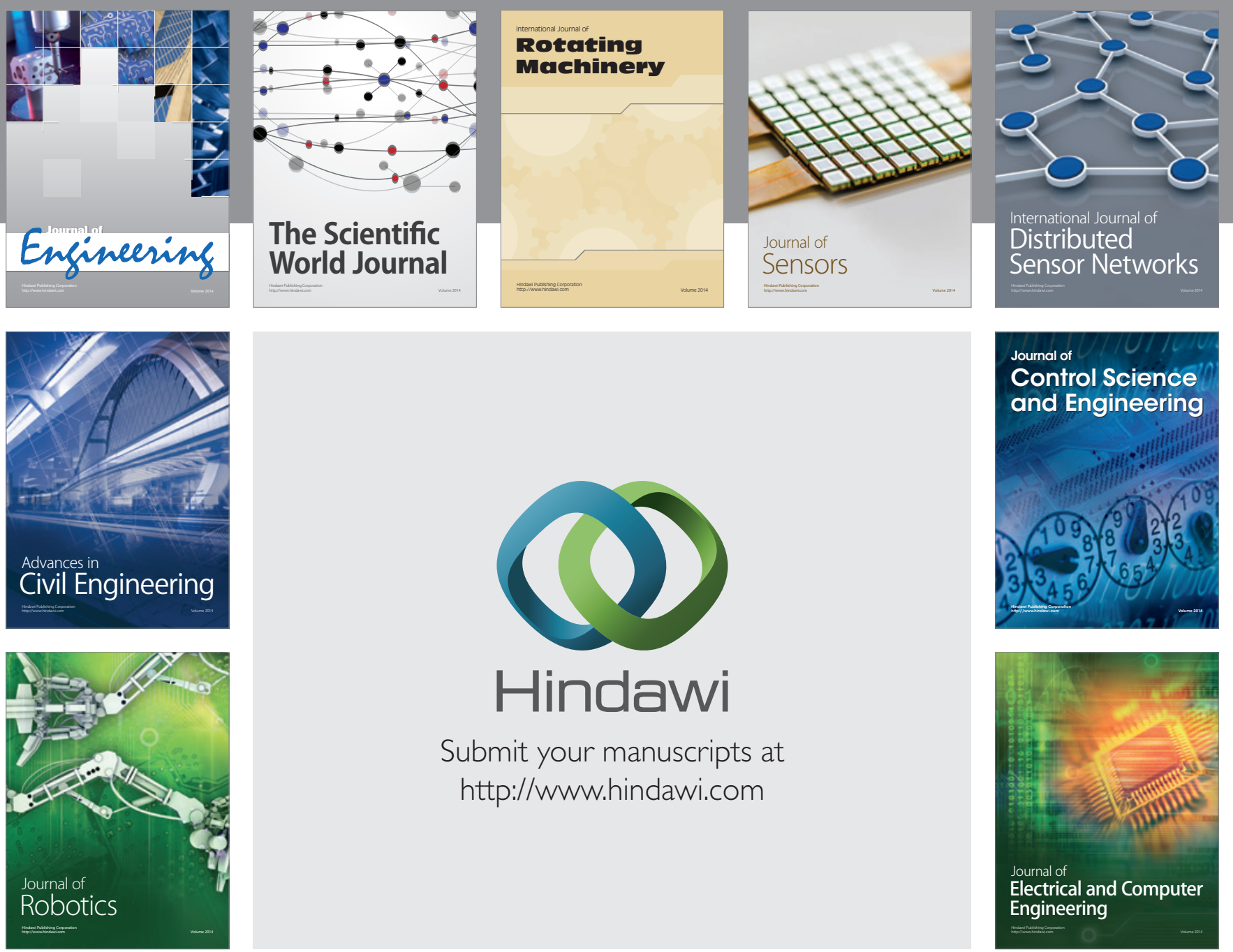

Submit your manuscripts at

http://www.hindawi.com
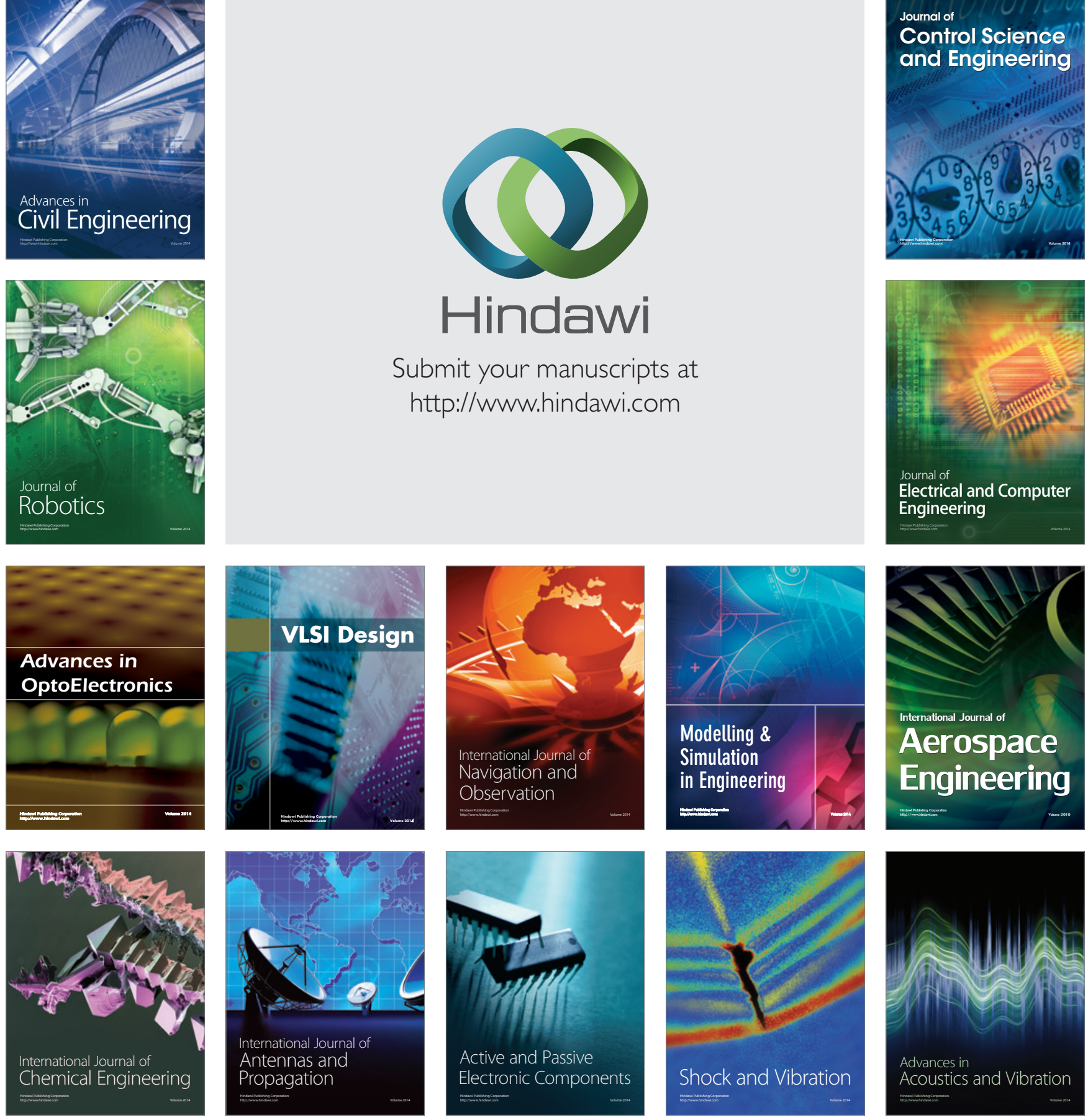